\title{
Correlative Microscopy Characterization of Cesium-Lead-Bromide Thin-films
}

\author{
Hannah Funk ${ }^{1 \dagger}$, Sebastian Caicedo-Davila ${ }^{1 \dagger}$, Robert Lovrincic ${ }^{2}$, Christian Müller ${ }^{2}$, Michael Sendner $^{3}$, \\ Frederike Lehmann ${ }^{1}$, René Gunder ${ }^{1}$, Alexandra Franz ${ }^{1}$, Markus Wollgarten ${ }^{1}$, Benedikt Haas ${ }^{4}$, Christoph T. \\ Koch $^{4}$, Daniel Abou-Ras ${ }^{1}$ \\ ${ }^{1}$ Helmholtz-Zentrum Berlin, Hahn-Meitner-Platz 1, 14109 Berlin, Germany. \\ ${ }^{2}$ InnovationLab GmbH, Speyerer Straße 4, 69115 Heidelberg, Germany. \\ ${ }^{3}$ Kirchhoff Institute of Physics, Heidelberg University, Heidelberg, Germany. \\ ${ }^{4}$ Humboldt-Universität zu Berlin, Unter den Linden 6, 10099 Berlin, Germany. \\ These authors contributed equally to this work.
}

\begin{abstract}
Inorganic cesium lead halide compounds have gained an increasing interest in the perovskite photovoltaics research community. These compounds are mixed into state of the art organic lead halide perovskite solar cells to provide for more thermal stability, and $\mathrm{CsPbX}_{3}(\mathrm{x}=\mathrm{I}, \mathrm{Br}, \mathrm{Cl})$ nanocubes are investigated as standalone emitter material in light emitting diodes. Eventually, reproducible, single-phase $\mathrm{CsPbBr}_{3}$ thin films could also provide us with a more stable inorganic material for perovskite solar cells. In the present work, we report on microscopic structural and optoelectronic properties of $\mathrm{Cs}-\mathrm{Pb}-\mathrm{Br}$ thin films prepared by different synthesis methods and studied using various electron-microscopy techniques.
\end{abstract}

Index Terms - cesium lead bromide, correlative microscopy, electron microscopy, halide Perovskites, phase distribution, secondary phases.

\section{INTRODUCTION}

The potential of metal-organic lead-halide perovskites as absorber materials for high-efficiency solar cells has attracted attention in recent years. Nevertheless, stability, reproducibility, and other limiting factors of the performance are still under investigation. Recently, inorganic cesium lead halides have gained an increasing interest in the perovskite research community. Mixing Cs into the perovskite absorber layer has been found to increase the thermal stability and reproducibility of organic lead halide solar cells [1]. Perovskite-like $\mathrm{CsPbX}_{3}$ (X=I, Br, Cl) have also been investigated as inorganic, potentially more stable, and wide-gap absorbers [2]. While the synthesis of $\mathrm{CsPbBr}_{3}$ with large crystals is well understood [3], and recent research on nanocrystalline $\mathrm{CsPbX}_{3}$ as active material in light emitting diodes achieve reproducible results [4], no study has compared the phase purity and reproducibility of polycrystalline $\mathrm{CsPbX}_{3}$ thin films deposition so far. Thus, the present work focuses on the study of secondary phases and their distributions within $\mathrm{Cs}-\mathrm{Pb}-\mathrm{Br}$ thin films synthesized by various methods.

The perovskite-like phase $\mathrm{CsPbBr}_{3}$ is subject to temperature-dependent phase transitions at $130^{\circ} \mathrm{C}$ from cubic to tetragonal and to orthorhombic at $88^{\circ} \mathrm{C}$. While the cubic "black" $\alpha$-phase corresponds to the perovskite-type $\mathrm{ABX}_{3}$ structure, the orthorhombic "yellow" $\delta$-phase at room temperature exhibits twice the number of atoms in the unit cell [5]. We hence call $\mathrm{CsPbBr}_{3}$ "perovskite-like". It is often referred to as monoclinic, owing to a slight deviation from the $90^{\circ}$ angle. Furthermore, depending on temperature and the stoichiometry, the ternary phases $\mathrm{CsPb}_{2} \mathrm{Br}_{5}$ and $\mathrm{Cs}_{4} \mathrm{PbBr}_{6}$ as well as the two binary phases $\mathrm{CsBr}$ and $\mathrm{PbBr}_{2}$ exist in addition to the perovskite like $\mathrm{CsPbBr}_{3}$ phase [6].

In the present contribution, we report characterization of $\mathrm{Cs}-\mathrm{Pb}-\mathrm{Br}$ thin films synthesized by spin-coating and coevaporation on two different substrates. We measured structural, compositional and optoelectronic properties in a correlative microscopy approach, combining various techniques.

\section{EXPERIMENTAL METHODS}

\section{A. Sample Preparation}

A $\mathrm{Cs} \mathrm{PbBr}_{3}$ powder sample was produced by wet chemical synthesis method. The spin-coated $\mathrm{Cs}-\mathrm{Pb}-\mathrm{Br}$ thin film on glass was prepared by a facile one-step solution method mixing the precursor $\mathrm{CsBr}$ with $\mathrm{PbBr}_{2}$ in a 1:1 molar ratio in a solvent before annealing. For the coevaporated thin films on glass and on a carbon-coated transmission electron microscope (TEM) $\mathrm{Cu}$-grid the precursors $\mathrm{PbBr}_{2}$ and $\mathrm{CsBr}$ where evaporated onto the substrate in a high vacuum setup for controlled fabrication of halide perovskite films. After deposition the films where annealed in $\mathrm{N}_{2}$ environment without exposition to ambient.

The thicknesses of the thin films was estimated by ellipsometry to $1 \mu \mathrm{m}$ for the spin-coated and coevaporated samples on glass as well as to $70 \mathrm{~nm}$ for the film on the TEM grid.

\section{B. Sample Analysis}

1) Structural analysis: X-ray diffraction (XRD) patterns where acquired with a Panalytical X'Pert Pro MPD diffractometer for thin film analysis with a $\mathrm{Cu}$-Anode $(\mathrm{K} \alpha 1+2)$. 
Bright field TEM images and electron diffraction patterns were acquired with a TEM Libra 200 microscope operating at $200 \mathrm{kV}$. The critical dose threshold for the coevaporated thin film was determined to be $5 \times 10^{20} \mathrm{e}^{-} / \mathrm{cm}^{2} \mathrm{~s}$ and hence condenser apertures down to $10 \mu \mathrm{m}$ were used to prevent sample degeneration.

For structural information diffraction patterns from $15 \times 15$ positions with a step size of $0.2 \mu \mathrm{m}$ were taken. An azimuthal integration of the intensity profiles of the superposition of the 255 diffraction patterns was performed with the ProcessDiffraction Software [7]. A camera length calibration was conducted using thallous chloride as diffraction standard.

Orientation-distribution maps were acquired with a transmission diffraction camera in a Zeiss Gemini 500 scanning electron microscope (SEM) at $30 \mathrm{kV}$ with a $7 \mu \mathrm{m}$ aperture.

2) Compositional analysis: Energy dispersive X-ray spectrometry (EDX) measurements were performed using a Zeiss UltraPlus SEM equipped with an Oxford Instruments XMax80 X-ray detector. Measurements were performed with a $10 \mathrm{kV}$ acceleration voltage and $60 \mu \mathrm{m}$ beam aperture. The pixel dwelling time for EDX mapping was set to $100 \mu$ s and EDX spectra were acquired until the net counts for the Cs- $\mathrm{L}$ peak reached 4 orders of magnitude. For quantification of stoichiometry, Cs-L, Pb-M and Br-L lines were used.

3) Optoelectronic Properties: Cathodoluminescence (CL) measurements were performed in a Zeiss MERLIN SEM using a SPARC system from Delmic, equipped with a Kymera 193i spectrograph and a Zyla 5.5 sCMOS camera from Andor. A monochromator grating of $300 \mathrm{l} / \mathrm{mm}$ blazed at $500 \mathrm{~nm}$ was used. The exposure time was set between 100 and $800 \mathrm{~ms}$ to ensure proper peak intensity. The acceleration voltage was set to $8 \mathrm{kV}$, and the beam current was $200 \mathrm{pA}$ for the spin-coated sample and $900 \mathrm{pA}$ for the evaporated ones. Photoluminescence (PL) spectra were measured using a 409 $\mathrm{nm}$ wavelength laser with a spot radius of $100 \mu \mathrm{m}$ at three different power setups: $0.3,1.0$ and $1.9 \mathrm{~W} / \mathrm{cm}^{2}$.

\section{Results AND Discussion}

For comparison with the XRD results (see Fig. 1) on the thin films, we used a $\mathrm{CsPbBr}_{3}$ powder sample, which exhibited an orthorhombic crystal structure with space group Pnam (62) [8]. While some of the perovskite phase peaks are not very prominent, the $\mathrm{Cs}-\mathrm{Pb}-\mathrm{Br}$ thin films exhibit additional peaks attributed to tetragonal $\mathrm{CsPb}_{2} \mathrm{Br}_{5}$ (space group $\mathrm{I} 4 / \mathrm{mcm}$ (140)) [14] and rhombohedral $\mathrm{Cs}_{4} \mathrm{PbBr}_{6}$ (space group $\mathrm{R}-3 \mathrm{c}$ (167)) [15]. Only the thin film of $70 \mathrm{~nm}$ thickness is in good agreement with the perovskite phase as far as the statistics from the very small volume allow to determine.

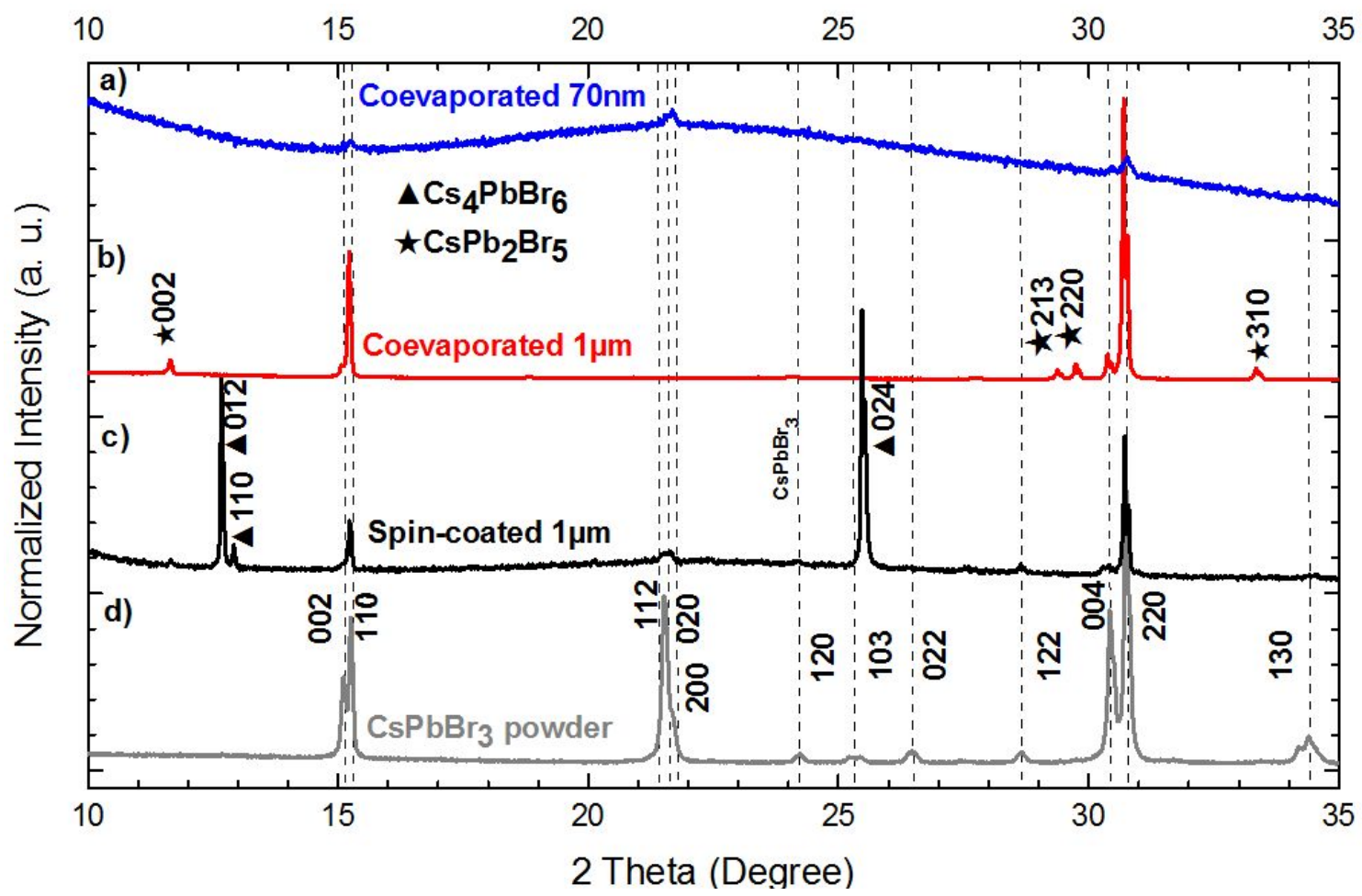

Fig. 1. XRD of the Cs-Pb-Pr thin films a) coevaporated (70nm) on Cu TEM-Grid, b) spin coated thin film on glass (1 $\mu \mathrm{m})$, c) coevaporated on glass $(1 \mu \mathrm{m})$ and $\mathrm{d})$ of the $\mathrm{CsPbX}_{3}$ powder reference. 


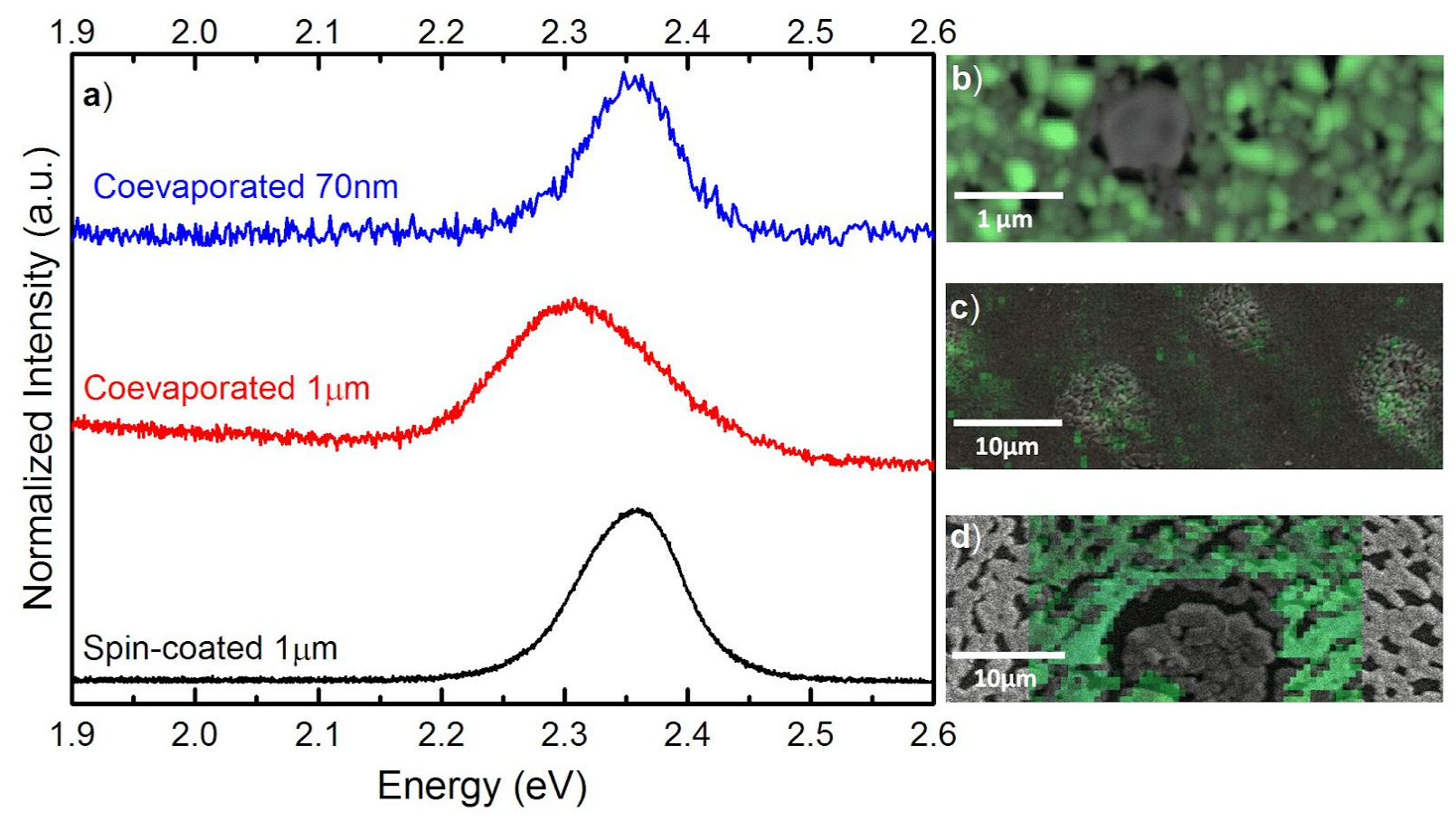

Fig. 2. a) CL spectra and the corresponding CL intensity maps, superimposed on the SEM images of the Cs-Pb-Br films b) coevaporated on a TEM grid, c) on glass, and d) spin-coated on glass.

\section{A. Coevaporated on Glass Substrate}

The XRD pattern of this sample is most similar to the $\mathrm{CsPbBr}_{3}$ powder reference, but is missing one of its most prominent peaks. Furthermore it displays peaks that can be assigned to $\mathrm{CsPb}_{2} \mathrm{Br}_{5}$.

We identified two different regions on this sample: a structured surface with regular defects apparently from mismatch of the crystals during the growth process, and a very flat surface. The PL response of the sample is very uneven throughout the surface. However we found regions that exhibited a strong peak at $2.35 \mathrm{eV}$, which corresponds well with the photoresponse reported in the literature for $\mathrm{CsPbBr}_{3}$ [9], [10]. CL maps also confirmed that the presence of luminescent peaks between 2.31 and $2.35 \mathrm{eV}$. The energy shift of the peaks at different pixels, which causes the broadening shown in Fig. 2-a, is caused by an artifact due to the large area scanned with the electron beam. EDX maps in Fig. 3 show an even distribution of $\mathrm{Cs}, \mathrm{Pb}$ and $\mathrm{Br}$ over the sample, both on the structured and flat regions.

\section{B. Spin-coated on Glass Substrate}

The XRD of this sample is dominated by peaks that can be attributed to $\mathrm{Cs}_{4} \mathrm{PbBr}_{6}$, but also exhibits peaks from the or possibly $\mathrm{CsPbBr}_{3}$ phase.
Two phases are distinguishable on this sample. Phase 1 is a highly luminescent porous matrix (see Fig. 2-d), while phase 2 is formed by precipitates of aggregated crystallites with no $\mathrm{CL}$ response in the visible spectral range. The integrated CL spectrum shows a peak at $2.35 \mathrm{eV}$, consistent with the bandgap transition of $\mathrm{CsPbBr}_{3}$ [9], and with the PL peak at $2.36 \mathrm{eV}$. Phase 1 is $\mathrm{Pb}$ depleted and $\mathrm{Cs}$ enhanced, while $\mathrm{Br}$ remained constant in both phases, as shown on the EDX maps in Fig. 3. We hypothesize that the phase 2 are either binary phases $\left(\mathrm{CsBr}\right.$ and $\left.\mathrm{PbBr}_{2}\right)$ or another ternary phase, which fits well with the measured optoelectronic properties, since they exhibit large gaps ( $>3 \mathrm{eV})$ [11]-[13].

\section{Coevaporated on $\mathrm{Cu}$-Grid}

Even though statistics for a film as thin as $70 \mathrm{~nm}$ are not very high the XRD diffraction diagram clearly displayed the main peaks of the $\mathrm{CsPbBr} 3$ phase.

With TEM imaging two types of regions could be defined on the sample: areas of a closed film with smaller grains $(50-100 \mathrm{~nm})$ and areas of a not closed film with larger grains $(100-500 \mathrm{~nm})$. Furthermore flat looking very large grains of sizes above $1 \mu \mathrm{m}$ where sprinkled throughout both areas. 

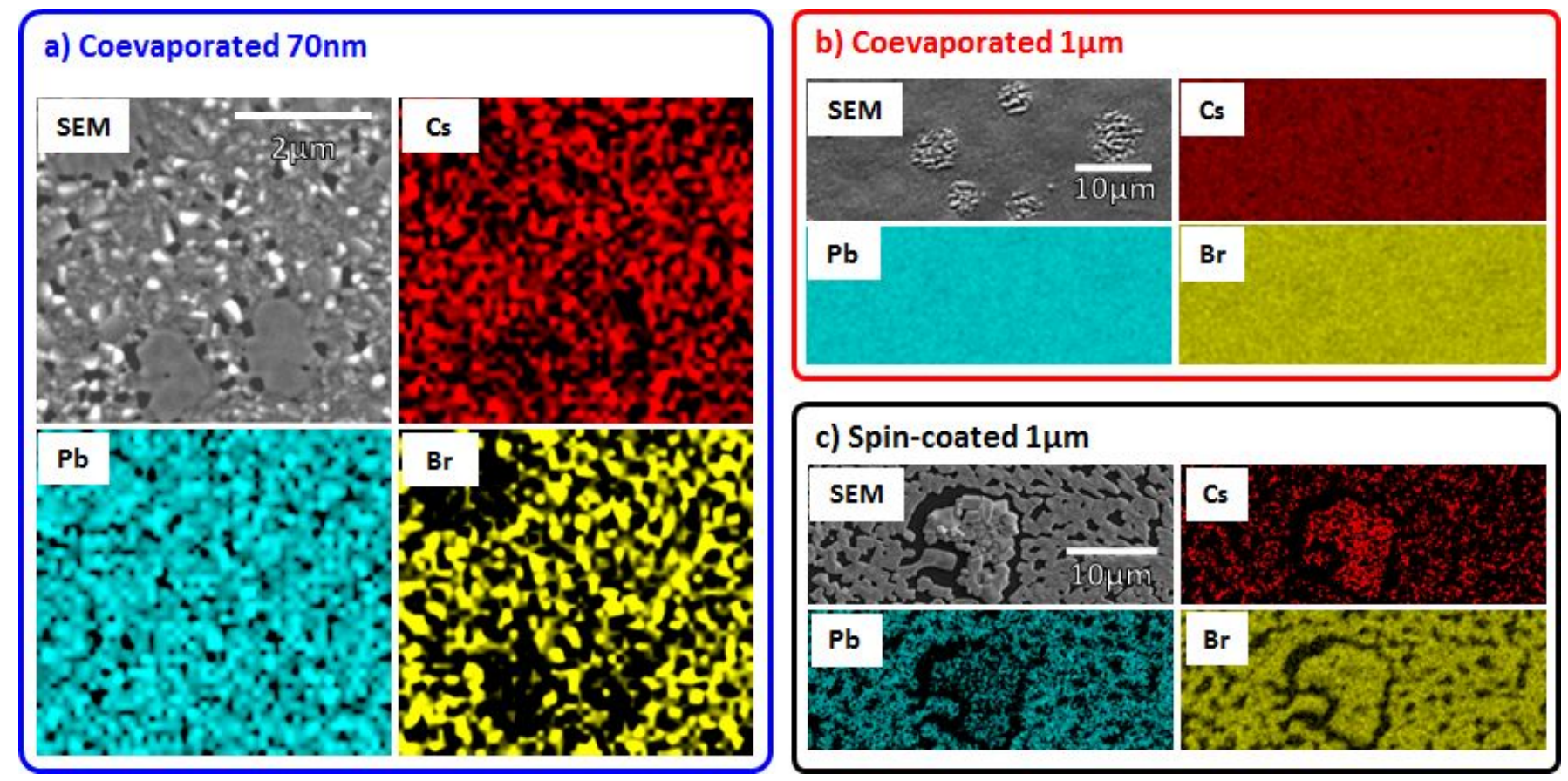

Fig. 3. SEM images and EDX maps, showing the elemental distribution on the Cs-Pb-Br films, coevaporated on a) TEM grid, b) glass, and c) -spin-coated on glass. The color maps show the intensity of the $\mathrm{Cs}, \mathrm{Pb}$ and $\mathrm{Br} \mathrm{X}$-ray peaks, corresponding to the element content in the film.

The structure information gathered from electron diffraction confirmed the main phase to be $\mathrm{CsPBr}_{3}$ for the closed as well as the not closed film. Nevertheless, both areas displayed slightly differing additional peaks stemming from secondary phases of $\mathrm{CsPb}_{2} \mathrm{Br}_{5}, \mathrm{Cs}_{4} \mathrm{PbBr}_{6}, \mathrm{CsBr}$ and $\mathrm{PbBr}_{2}$.

With orientation-distribution maps the smaller and larger grains could be shown to be $\mathrm{CsPbBr}_{3}$ while the very large grains could be identified as $\mathrm{CsPb}_{2} \mathrm{Br}_{5}$ (see Fig. 4). This could be confirmed with the relative stoichiometry obtained from EDX measurements (see Fig. 3-a).

The CL mapping confirmed the phase distribution. The accumulated CL spectrum for the closed film region exhibits an individual peak at $2.36 \mathrm{eV}$, as detected for phase 1 of the spin-coated sample. From th CL mapping, the grains forming the film were to be found highly luminescent while the large grains showed no luminescence in the measured visible range. This is in agreement with the STEM experiment identifying the large grains as $\mathrm{CsPb}_{2} \mathrm{Br}_{5}$ and the matrix grains as $\mathrm{CsPbBr}_{3}$.

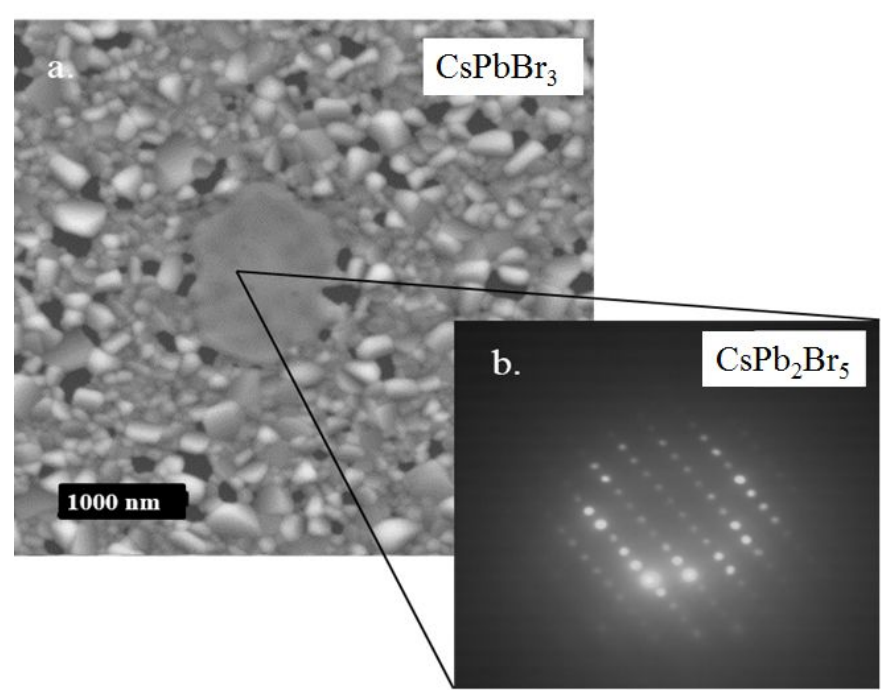

Fig. 4. SEM image of the coevaporated sample on a $\mathrm{Cu}$-grid showing a) closed film with mostly small grains with one very large grain. b) Transmission electron diffraction pattern of a very large grain identified as $\mathrm{CsPb}_{2} \mathrm{Br}_{5}$. 


\section{CONCLUSION}

We measured structural, compositional, and optoelectronic properties of $\mathrm{Cs}-\mathrm{Pb}-\mathrm{Br}$ thin films synthesized by spin-coating and coevaporation at various scales by means of XRD, PL spectroscopy as well as SEM and TEM analysis. This correlative work shows that obtaining a single-phase $\mathrm{CsPbBr}_{3}$ film is not a trivial task and depends on the synthesis method and on a careful control of the growth parameters. In contrast to $\mathrm{CsPbBr} 3$ powder, the $\mathrm{Cs}-\mathrm{Pb}-\mathrm{Br}$ thin films exhibit phases secondary to $\mathrm{CsPbBr}_{3}$ - such as $\mathrm{CsPb}_{2} \mathrm{Br}_{5}, \mathrm{Cs}_{4} \mathrm{PbBr}_{6}$ - and residuals of the binary compounds $\mathrm{CsBr}$ and $\mathrm{PbBr}_{2}$. Spin-coated films on glass feature lateral distributions of secondary phases on the $\mu \mathrm{m}$ scale and are mainly composed of $\mathrm{CsPbBr}_{3}$ and $\mathrm{Cs}_{4} \mathrm{PbBr}_{6}$, which indicates $\mathrm{CsBr}$ excess. On the other hand, coevaporated films are a mixture of the $\mathrm{CsPbBr}_{3}$ and $\mathrm{CsPb}_{2} \mathrm{Br}_{5}$ phases, indicating a $\mathrm{PbBr}$ excess during the deposition. A better control of the coevaporation leads to a $\mathrm{CsPbBr}_{3}$ film accompanied by secondary phases on the scale of hundreds of nm. Further research on $\mathrm{Cs}-\mathrm{Pb}-\mathrm{Br}$ is necessary to improve the reproducibility of the different synthesis methods.

\section{ACKNOWLEDGEMENT}

The authors are grateful for financial support by HyPerCell Graduate School, the Helmholtz International Research School HI-SCORE and the DFG for funding the BerlinEM Network core facility (DFG grant no. KO 2911/12-1). Special thanks are due to Ulrike Bloeck, HZB, for continuous support by specimen preparation.

\section{REFERENCES}

[1] M. Saliba et al., "Cesium-containing triple cation perovskite solar cells: improved stability, reproducibility and high efficiency," Energy Environ. Sci., vol. 9, no. 6, pp. 1989-1997, 2016.

[2] M. Kulbak, D. Cahen, and G. Hodes, "How Important Is the Organic Part of Lead Halide Perovskite Photovoltaic Cells? Efficient $\mathrm{CsPbBr}_{3}$ Cells,” J. Phys. Chem. Lett., vol. 6, no. 13, pp. 2452-2456, 2015.

[3] Y. Rakita et al., "Low-Temperature Solution-Grown $\mathrm{CsPbBr}$ Single Crystals and Their Characterization," Cryst. Growth Des., vol. 16, no. 10, pp. 5717-5725, 2016.

[4] M. Chen et al., "Solvothermal Synthesis of High-Quality All-Inorganic Cesium Lead Halide Perovskite Nanocrystals: From Nanocube to Ultrathin Nanowire," Adv. Funct. Mater., vol. 27 , no. $23,2017$.

[5] C. K. Moller, "The Structure Of Perovskite-Like Cæsium Plumbo Trihalides," Mat. Fys. Medd. Dan. Vid. Selsk, vol. 32, no. 2, 1959.

[6] M. Cola and R. Riccardi, "Binary Systems Formed by Lead Bromide with ( $\mathrm{Li}, \mathrm{Na}, \mathrm{K}, \mathrm{Rb}, \mathrm{Cs}$ and $\mathrm{Tl}$ ) $\mathrm{Br}$ : a DTA and Diffractometric Study," Zeitschrift für Naturforsch. A, vol. 26, p. $1328,1971$.
[7] J. L. Lábár, "Microscopy Microanalysis Electron Diffraction Based Analysis of Phase Fractions and Texture in Nanocrystalline Thin Films , Part I : Principles," pp. 20-29, 2008.

[8] C. C. Stoumpos et al., "Crystal Growth of the Perovskite Semiconductor $\mathrm{CsPbBr}_{3}$ : A New Material for High-Energy Radiation Detection," Cryst. Growth Des., vol. 13, p. 2722-2727, 2013.

[9] M. I. Saidaminov et al., "Pure $\mathrm{Cs}_{4} \mathrm{PbBr}_{6}$ : Highly Luminescent Zero-Dimensional Perovskite Solids," ACS Energy Lett., vol. 1, no. 4, pp. 840-845, 2016.

[10] J.-H. H. Cha et al., "Photoresponse of $\mathrm{CsPbBr}_{3}$ and $\mathrm{Cs}_{4} \mathrm{PbBr}_{6}$ Perovskite Single Crystals," J. Phys. Chem. Lett., vol. 8, no. 3, pp. 565-570, 2017.

[11] A. J. H. Eijkelenkamp, "Photoluminescence of $\mathrm{PbBr}_{2}, \mathrm{PbCl}_{2}$ and $\beta-\mathrm{PbF}_{2}$ single crystals," J. Lumin., vol. 15 , no. 2, pp. 217-225, 1977.

[12] J. L. Jansons, V. J. Krumins, Z. A. Rachko, and J. A. Valbis, "Luminescence Due to Radiative Transitions between Valence Band and Upper Core Band in Ionic Crystals (Crossluminescence)," Phys. Status Solidi, vol. 144, no. 2, pp. 835-844, 1987.

[13] S. Kubota, J. zhi Ruan (Gen), M. Itoh, S. Hashimoto, and S. Sakuragi, "A new type of luminescence mechanism in large band-gap insulators: Proposal for fast scintillation materials," Nucl. Inst. Methods Phys. Res. A, vol. 289, no. 1-2, pp. 253-260, 1990.

[14] Z. Zhang, Y. Zhu, W. Wang, W. Zheng, R. Lin, and F. Huang, "Growth, characterization and optoelectronic applications of pure-phase large-area $\mathrm{CsPb}_{2} \mathrm{Br}_{5}$ flake single crystals," J. Mater. Chem. C, vol. 6, no. 3, pp. 446-451, 2018.

[15] R. H. Andrews, S. J. Clark, J. D. Donaldson, J. C. Dewan, and J. Silver, "Solid-state properties of materials of the type $\mathrm{Cs}_{4} \mathrm{MX}_{6}$ (where $\mathrm{M}=\mathrm{Sn}$ or $\mathrm{Pb}$ and $\mathrm{X}=\mathrm{Cl}$ or $\mathrm{Br}$ )," J. Chem. Soc. Dalt. Trans., no. 4, p. 767, 1983. 